\title{
Clique-Width for Graph Classes Closed under Complementation*
}

\author{
Alexandre Blanché ${ }^{1}$, Konrad K. Dabrowski ${ }^{2}$, Matthew Johnson ${ }^{3}$, \\ Vadim V. Lozin ${ }^{4}$, Daniël Paulusma ${ }^{5}$, and Viktor Zamaraev ${ }^{6}$ \\ 1 École normale supérieure de Rennes, Rennes, France \\ alexandre.blanche@ens-rennes.fr \\ 2 Durham University, Durham, UK \\ konrad.dabrowski@durham.ac.uk \\ 3 Durham University, Durham, UK \\ matthew.johnson2@durham.ac.uk \\ 4 University of Warwick, Coventry, UK \\ v.lozin@warwick.ac.uk \\ 5 Durham University, Durham, UK \\ daniel.paulusma@durham.ac.uk \\ 6 University of Warwick, Coventry, UK \\ v.zamaraev@warwick.ac.uk
}

\begin{abstract}
Clique-width is an important graph parameter due to its algorithmic and structural properties. A graph class is hereditary if it can be characterized by a (not necessarily finite) set $\mathcal{H}$ of forbidden induced subgraphs. We initiate a systematic study into the boundedness of cliquewidth of hereditary graph classes closed under complementation. First, we extend the known classification for the $|\mathcal{H}|=1$ case by classifying the boundedness of clique-width for every set $\mathcal{H}$ of self-complementary graphs. We then completely settle the $|\mathcal{H}|=2$ case. In particular, we determine one new class of $(H, \bar{H})$-free graphs of bounded clique-width (as a side effect, this leaves only six classes of $\left(H_{1}, H_{2}\right)$-free graphs, for which it is not known whether their cliquewidth is bounded). Once we have obtained the classification of the $|\mathcal{H}|=2$ case, we research the effect of forbidding self-complementary graphs on the boundedness of clique-width. Surprisingly, we show that for a set $\mathcal{F}$ of self-complementary graphs on at least five vertices, the classification of the boundedness of clique-width for $(\{H, \bar{H}\} \cup \mathcal{F})$-free graphs coincides with the one for the $|\mathcal{H}|=2$ case if and only if $\mathcal{F}$ does not include the bull (the only non-empty self-complementary graphs on fewer than five vertices are $P_{1}$ and $P_{4}$, and $P_{4}$-free graphs have clique-width at most 2). Finally, we discuss the consequences of our results for Colouring.
\end{abstract}

1998 ACM Subject Classification G.2.2 Graph Theory

Keywords and phrases clique-width, self-complementary graph, forbidden induced subgraph

Digital Object Identifier 10.4230/LIPIcs.MFCS.2017.73

\section{Introduction}

Many graph-theoretic problems that are computationally hard for general graphs may still be solvable in polynomial time if the input graph can be decomposed into large parts of "similarly

\footnotetext{
* This paper received support from EPSRC (EP/K025090/1 and EP/L020408/1) and the Leverhulme Trust (RPG-2016-258).

(c) (i) Alexandre Blanché, Konrad K. Dabrowski, Matthew Johnson, Vadim V. Lozin, Daniël Paulusma, and Viktor Zamaraev;

licensed under Creative Commons License CC-BY

42nd International Symposium on Mathematical Foundations of Computer Science (MFCS 2017).

Editors: Kim G. Larsen, Hans L. Bodlaender, and Jean-Francois Raskin; Article No. 73; pp. 73:1-73:14

Leibniz International Proceedings in Informatics

LIPICS Schloss Dagstuhl - Leibniz-Zentrum für Informatik, Dagstuhl Publishing, Germany
} 
behaving" vertices. Such decompositions may lead to an algorithmic speed up and are often defined via some type of graph construction. One particular type is to use vertex labels and to allow certain graph operations, which ensure that vertices labelled alike will always keep the same label and thus behave identically. The clique-width cw $(G)$ of a graph $G$ is the minimum number of different labels needed to construct $G$ using four such operations (see Section 2 for details). Clique-width has been studied extensively both in algorithmic and structural graph theory. The main reason for its popularity is that, indeed, many well-known NP-hard problems $[14,25,35,40]$, such as Colouring and Hamilton Cycle, become polynomialtime solvable on any graph class $\mathcal{G}$ of bounded clique-width, that is, for which there exists a constant $c$, such that every graph in $\mathcal{G}$ has clique-width at most $c$. Graph Isomorphism is also polynomial-time solvable on such graph classes [30]. Having bounded clique-width is equivalent to having bounded rank-width [39] and having bounded NLC-width [33], two other well-known width-parameters. However, despite these close relationships, clique-width is a notoriously difficult graph parameter, and our understanding of it is still very limited. For instance, no polynomial-time algorithms are known for computing the clique-width of very restricted graph classes, such as unit interval graphs, or for deciding whether a graph has clique-width at most $4 .{ }^{1}$ In order to get a better understanding of clique-width and to identify new "islands of tractability" for central NP-hard problems, many graph classes of bounded and unbounded clique-width have been identified; see, for instance, the Information System on Graph Classes and their Inclusions [24], which keeps a record of such graph classes. In this paper we study the following research question:

\section{What kinds of properties of a graph class ensure that its clique-width is bounded?}

We refer to the surveys $[31,34]$ for examples of such properties. Here, we consider graph complements. The complement $\bar{G}$ of a graph $G$ is the graph with vertex set $V_{G}$ and edge set $\{u v \mid u v \notin E(G)\}$ and has clique-width $\operatorname{cw}(\bar{G}) \leq 2 \mathrm{cw}(G)[15]$. This result implies that a graph class $\mathcal{G}$ has bounded clique-width if and only if the class consisting of all complements of graphs in $\mathcal{G}$ has bounded clique-width. Due to this, we initiate a systematic study of the boundedness of clique-width for graph classes $\mathcal{G}$ closed under complementation, that is, for every graph $G \in \mathcal{G}$, its complement $\bar{G}$ also belongs to $\mathcal{G}$.

To get a handle on graph classes closed under complementation, we restrict ourselves to graph classes that are not only closed under complementation but also under vertex deletion. This is a natural assumption, as deleting a vertex does not increase the clique-width of a graph. A graph class closed under vertex deletion is said to be hereditary and can be characterized by a (not necessarily finite) set $\mathcal{H}$ of forbidden induced subgraphs. Over the years many results on the (un)boundedness of clique-width of hereditary graph classes have appeared. We briefly survey some of these results below.

A hereditary graph class of graphs is monogenic or $H$-free if it can be characterized by one forbidden induced subgraph $H$, and bigenic or $\left(H_{1}, H_{2}\right)$-free if it can be characterized by two forbidden induced subgraphs $H_{1}$ and $H_{2}$. It is well known (see [23]) that a class of $H$-free graphs has bounded clique-width if and only if $H$ is an induced subgraph of $P_{4}{ }^{2}$ By combining known results $[3,5,7,8,9,10,11,17,18,21,38]$ with new results for bigenic graph classes, Dabrowski and Paulusma [23] classified the (un)boundedness of clique-width of $\left(H_{1}, H_{2}\right)$-free graphs for all but 13 pairs $\left(H_{1}, H_{2}\right)$ (up to an equivalence relation). Afterwards,

\footnotetext{
1 It is known that computing clique-width is NP-hard in general [27] and that deciding whether a graph has clique-width at most 3 is polynomial-time solvable [13].

2 We refer to Section 2 for all the notation used in this section.
} 


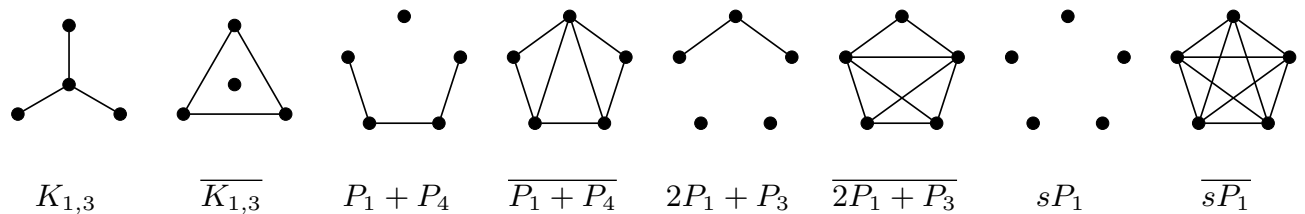

Figure 1 Graphs $H$ for which the clique-width of $(H, \bar{H})$-free graphs is bounded ( $s=5$ is shown).

five new classes of $\left(H_{1}, H_{2}\right)$-free graphs were identified by Dross et al. [16] and recently, another one was identified by Dabrowski et al. [19]. This means that only seven cases $\left(H_{1}, H_{2}\right)$ remained open. Other systematic studies were performed for $H$-free weakly chordal graphs [5], $H$-free chordal graphs [5] (two open cases), $H$-free triangle-free graphs [19] (two open cases), $H$-free bipartite graphs [22], $H$-free split graphs [4] (two open cases), and $\mathcal{H}$-free graphs where $\mathcal{H}$ is any set of 1 -vertex extensions of the $P_{4}[6]$ or any set of graphs on at most four vertices [7]. Clique-width results or techniques for these graph classes impacted upon each other and could also be used for obtaining new results for bigenic graph classes.

Our Contribution. Recall that we investigate the clique-width of hereditary graph classes closed under complementation. A graph that contains no induced subgraph isomorphic to a graph in a set $\mathcal{H}$ is said to be $\mathcal{H}$-free. We first consider the $|\mathcal{H}|=1$ case. The class of $H$-free graphs is closed under complementation if and only if $H$ is a self-complementary graph, that is, $H=\bar{H}$. Self-complementary graphs have been extensively studied; see [26] for a survey. From the aforementioned result for $P_{4}$-free graphs, we find that the only self-complementary graphs $H$ for which the class of $H$-free graphs has bounded clique-width are $H=P_{1}$ and $H=P_{4}$. In Section 3 we prove the following generalization of this result.

- Theorem 1. Let $\mathcal{H}$ be a set of non-empty self-complementary graphs. Then the class of $\mathcal{H}$-free graphs has bounded clique-width if and only if either $P_{1} \in \mathcal{H}$ or $P_{4} \in \mathcal{H}$.

We now consider the $|\mathcal{H}|=2$ case. Let $\mathcal{H}=\left\{H_{1}, H_{2}\right\}$. Due to Theorem 1 we may assume $H_{2}=\overline{H_{1}}$ and $H_{1}$ is not self-complementary. The class of $\left(2 P_{1}+P_{3}, \overline{2 P_{1}+P_{3}}\right)$-free graphs was one of the seven remaining bigenic graph classes, and the only bigenic graph class closed under complementation, for which boundedness of clique-width was open. We settle this case by proving in Section 4 that the clique-width of this class is bounded. In the same section we combine this new result with known results to prove the following theorem, which, together with Theorem 1, shows to what extent the property of being closed under complementation helps with bounding the clique-width for bigenic graph classes (see also Figure 1).

- Theorem 2. For a graph $H$, the class of $(H, \bar{H})$-free graphs has bounded clique-width if and only if $H$ or $\bar{H}$ is an induced subgraph of $K_{1,3}, P_{1}+P_{4}, 2 P_{1}+P_{3}$ or $s P_{1}$ for some $s \geq 1$.

For the $|\mathcal{H}|=3$ case, where $\left\{H_{1}, H_{2}, H_{3}\right\}=\mathcal{H}$, we observe that a class of $\left(H_{1}, H_{2}, H_{3}\right)$-free graphs is closed under complementation if and only if either every $H_{i}$ is self-complementary, or one $H_{i}$ is self-complementary and the other two graphs $H_{j}$ and $H_{k}$ are complements of each other. By Theorem 1, we only need to consider $\left(H_{1}, \overline{H_{1}}, H_{2}\right)$-free graphs, where $H_{1}$ is not self-complementary, $H_{2}$ is self-complementary, and neither $H_{1}$ nor $H_{2}$ is an induced subgraph of $P_{4}$. The next two smallest self-complementary graphs $H_{2}$ are the $C_{5}$ and the bull (see also Figure 2). Observe that any self-complementary graph on $n$ vertices must contain $\frac{1}{2}\left(\begin{array}{c}n \\ 2\end{array}\right)$ edges and this number must be an integer, so $n=4 q$ or $n=4 q+1$ for some integer $q \geq 0$. There are exactly ten non-isomorphic self-complementary graphs on eight vertices [41] and we depict these in Figure 3. 


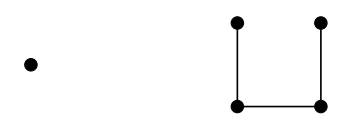

$P_{1}$

$P_{4}$

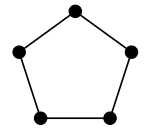

$C_{5}$

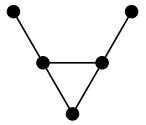

bull

Figure 2 The four non-empty self-complementary graphs on less than eight vertices [41].
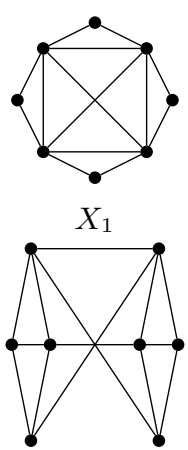

$X_{6}$
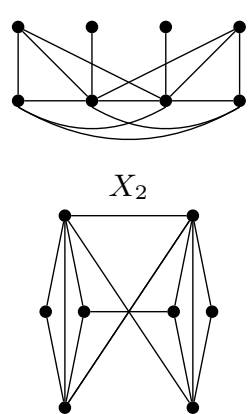

$X_{7}$

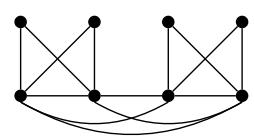

$X_{3}$

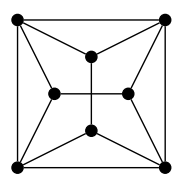

$X_{8}$

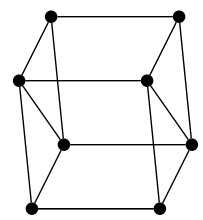

$X_{4}$

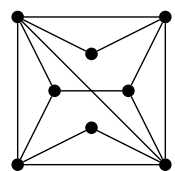

$X_{9}$

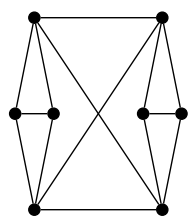

$X_{5}$

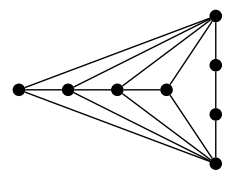

$X_{10}$

Figure 3 The ten self-complementary graphs on eight vertices [41].

It is known that split graphs, or equivalently, $\left(2 P_{2}, \overline{2 P_{2}}, C_{5}\right)$-free graphs have unbounded clique-width [38]. In Section 5 we determine three new hereditary graph classes of unbounded clique-width, which imply that the class of $\left(H, \bar{H}, C_{5}\right)$-free graphs has unbounded clique-width if $H \in\left\{K_{1,3}+P_{1}, 2 P_{2}, 3 P_{1}+P_{2}, S_{1,1,2}\right\}$. By combining this with known results, we discovered that the classification of boundedness of clique-width for $\left(H, \bar{H}, C_{5}\right)$-free graphs coincides with the one of Theorem 2. This raised the question of whether the same is true for other sets of self-complementary graphs $\mathcal{F} \neq\left\{C_{5}\right\}$. If $\mathcal{F}$ contains the bull, then the answer is negative: by Theorem 2 , the class of $\left(S_{1,1,2}, \overline{S_{1,1,2}}\right)$-free graphs and the class of $\left(2 P_{2}, C_{4}\right)$-free graphs both have unbounded clique-width, but both the class of $\left(S_{1,1,2}, \overline{S_{1,1,2}}\right.$, bull)-free graphs and even the class of $\left(P_{5}, \overline{P_{5}}\right.$, bull)-free graphs have bounded clique-width [6]. However, also in Section 5, we prove that the bull is the only exception (apart from the trivial cases when $H^{\prime} \in\left\{P_{1}, P_{4}\right\}$ which yield bounded clique-width of $\left(H, \bar{H}, H^{\prime}\right)$-free graphs for any graph $\left.H\right)$.

- Theorem 3. Let $\mathcal{F}$ be a set of self-complementary graphs on at least five vertices not equal to the bull. For a graph $H$, the class of $(\{H, \bar{H}\} \cup \mathcal{F})$-free graphs has bounded clique-width if and only if $H$ or $\bar{H}$ is an induced subgraph of $K_{1,3}, P_{1}+P_{4}, 2 P_{1}+P_{3}$ or $s P_{1}$ for some $s \geq 1$.

Consequences. Due to our result for $\left(2 P_{1}+P_{3}, \overline{2 P_{1}+P_{3}}\right)$-free graphs, we can update the summary of [19] for the clique-width of bigenic graph classes and reduce the number of open cases from seven to six.

- Open Problem 4. Have $\left(H_{1}, H_{2}\right)$-free graphs bounded or unbounded clique-width when:

(i) $H_{1}=3 P_{1}$ and $\overline{H_{2}} \in\left\{P_{1}+S_{1,1,3}, S_{1,2,3}\right\}$;

(ii) $H_{1}=2 P_{1}+P_{2}$ and $\overline{H_{2}} \in\left\{P_{1}+P_{2}+P_{3}, P_{1}+P_{5}\right\}$;

(iii) $H_{1}=P_{1}+P_{4}$ and $\overline{H_{2}} \in\left\{P_{1}+2 P_{2}, P_{2}+P_{3}\right\}$. 
Another consequence of our result for $\left(2 P_{1}+P_{3}, \overline{2 P_{1}+P_{3}}\right)$-free graphs is that Colouring is polynomial-time solvable for this graph class. This result was used by Blanché et al. [2]:

- Theorem 5 ([2]). Let $H, \bar{H} \notin\left\{(s+1) P_{1}+P_{3}, s P_{1}+P_{4} \mid s \geq 2\right\}$. Then Colouring is polynomial-time solvable for $(H, \bar{H})$-free graphs if $H$ or $\bar{H}$ is an induced subgraph of $K_{1,3}$, $P_{1}+P_{4}, 2 P_{1}+P_{3}, P_{2}+P_{3}, P_{5}$, or $s P_{1}+P_{2}$ for some $s \geq 0$ and it is NP-complete otherwise.

Comparing Theorems 2 and 5 shows that there are graph classes of unbounded cliquewidth closed under complementation for which COLOURING is polynomial-time solvable. Nevertheless, on many graph classes, polynomial-time solvability of NP-hard problems stems from the underlying property of having bounded clique-width. The present paper illustrates this for the Colouring problem, since Theorem 28 implies that Colouring is solvable in polynomial time on $\left(2 P_{1}+P_{3}, \overline{2 P_{1}+P_{3}}\right)$-free graphs. By updating the summary of [16] (see also [29]), we find that there are twelve classes of $\left(H_{1}, H_{2}\right)$-free graphs, for which CoLOURING could still potentially be solved in polynomial time by showing that their clique-width is bounded.

Future Work. Apart from settling the classification of boundedness of clique-width for $\left(H_{1}, H_{2}\right)$-free graphs by addressing Open Problem 4, we aim to continue our study of boundedness of clique-width for graph classes closed under complementation. In particular, to complete the classification for $\mathcal{H}$-free graphs when $|\mathcal{H}|=3$, we still need to determine those graphs $H$ for which $(H, \bar{H}$, bull)-free graphs have bounded clique-width (there are several cases left).

\section{Preliminaries}

The disjoint union $(V(G) \cup V(H), E(G) \cup E(H))$ of two vertex-disjoint graphs $G$ and $H$ is denoted by $G+H$ and the disjoint union of $r$ copies of a graph $G$ is denoted by $r G$. For a subset $S \subseteq V(G)$, we let $G[S]$ denote the subgraph of $G$ induced by $S$. If $S=\left\{s_{1}, \ldots, s_{r}\right\}$ then, to simplify notation, we may also write $G\left[s_{1}, \ldots, s_{r}\right]$ instead of $G\left[\left\{s_{1}, \ldots, s_{r}\right\}\right]$. We write $G \backslash S=G[V(G) \backslash S]$; if $S=\{v\}$, we may write $G \backslash v$ instead. We write $G^{\prime} \subseteq_{i} G$ to indicate that $G^{\prime}$ is an induced subgraph of $G$. The graphs $C_{r}, K_{r}, K_{1, r-1}$ and $P_{r}$ denote the cycle, complete graph, star and path on $r$ vertices, respectively. The graphs $K_{3}$ and $K_{1,3}$ are also called the triangle and claw. The graph $S_{h, i, j}$, for $1 \leq h \leq i \leq j$, denotes the subdivided claw, that is, the tree with only one vertex $x$ of degree 3 and exactly three leaves, which are of distance $h, i$ and $j$ from $x$, respectively. Observe $S_{1,1,1}=K_{1,3}$. We let $\mathcal{S}$ be the class of graphs each connected component of which is either a subdivided claw or a path.

For a set of graphs $\mathcal{H}$, a graph $G$ is $\mathcal{H}$-free (or $(\mathcal{H})$-free) if it has no induced subgraph isomorphic to a graph in $\mathcal{H}$. If $\mathcal{H}=\left\{H_{1}, \ldots, H_{p}\right\}$ for some integer $p$, then we may write $\left(H_{1}, \ldots, H_{p}\right)$-free instead of $\left(\left\{H_{1}, \ldots, H_{p}\right\}\right)$-free, or, if $p=1$, we may simply write $H_{1}$-free. For a graph $G=(V, E)$, the set $N(u)=\{v \in V \mid u v \in E\}$ denotes the neighbourhood of $u \in V$. A graph is bipartite if its vertex set can be partitioned into two (possibly empty) independent sets. A graph is split if its vertex set can be partitioned into a (possibly empty) independent set and a (possibly empty) clique. Split graphs have been characterized as follows.

- Lemma 6 ([28]). A graph $G$ is split if and only if it is $\left(2 P_{2}, C_{4}, C_{5}\right)$-free.

Let $X$ be a set of vertices in a graph $G=(V, E)$. A vertex $y \in V \backslash X$ is complete to $X$ if it is adjacent to every vertex of $X$ and anti-complete to $X$ if it is non-adjacent to every 
vertex of $X$. Similarly, a set of vertices $Y \subseteq V \backslash X$ is complete (resp. anti-complete) to $X$ if every vertex in $Y$ is complete (resp. anti-complete) to $X$. We say that the edges between two disjoint sets of vertices $X$ and $Y$ form a matching (resp. co-matching) if each vertex in $X$ has at most one neighbour (resp. non-neighbour) in $Y$ and vice versa (if each vertex has exactly one such neighbour, we say that the matching is perfect). A vertex $y \in V \backslash X$ distinguishes $X$ if $y$ has both a neighbour and a non-neighbour in $X$. The set $X$ is a module of $G$ if no vertex in $V \backslash X$ distinguishes $X$. A module $X$ is non-trivial if $1<|X|<|V|$, otherwise it is trivial. A graph is prime if it has only trivial modules.

To help reduce the amount of case analysis needed to prove Theorems 2 and 3, we will use the following lemma (proof omitted).

- Lemma 7. Let $H \in \mathcal{S}$. Then $H$ is $\left(K_{1,3}+P_{1}, 2 P_{2}, 3 P_{1}+P_{2}, S_{1,1,2}\right)$-free if and only if $H$ is an induced subgraph of $K_{1,3}, P_{1}+P_{4}, 2 P_{1}+P_{3}$ or $s P_{1}$ for some $s \geq 1$.

The clique-width $\operatorname{cw}(G)$ of a graph $G$ is the minimum number of labels needed to construct $G$ by using the following four operations:

1. creating a new graph consisting of a single vertex $v$ with label $i$;

2. taking the disjoint union of two labelled graphs $G_{1}$ and $G_{2}$;

3. joining each vertex with label $i$ to each vertex with label $j(i \neq j)$;

4. renaming label $i$ to $j$.

For an induced subgraph $G^{\prime}$ (or vertex set $X \subseteq V(G)$ ) of a graph $G$, the subgraph complementation operation replaces every edge present in $G^{\prime}$ (resp. $G[X]$ ) by a non-edge, and vice versa. For two disjoint vertex subsets $S$ and $T$ in $G$, the bipartite complementation operation replaces every edge with one end-vertex in $S$ and the other one in $T$ by a non-edge and vice versa. Let $k \geq 0$ be a constant and let $\gamma$ be some graph operation. A class $\mathcal{G}^{\prime}$ is $(k, \gamma)$-obtained from a class $\mathcal{G}$ if:

1. every graph in $\mathcal{G}^{\prime}$ is obtained from a graph in $\mathcal{G}$ by performing $\gamma$ at most $k$ times, and

2. for every $G \in \mathcal{G}$ there exists at least one graph in $\mathcal{G}^{\prime}$ that is obtained from $G$ by performing $\gamma$ at most $k$ times.

We say that $\gamma$ preserves boundedness of clique-width if for any finite constant $k$ and any graph class $\mathcal{G}$, any graph class $\mathcal{G}^{\prime}$ that is $(k, \gamma)$-obtained from $\mathcal{G}$ has bounded clique-width if and only if $\mathcal{G}$ has bounded clique-width.

Fact 1. Vertex deletion preserves boundedness of clique-width [36].

Fact 2. Subgraph complementation preserves boundedness of clique-width [34].

Fact 3. Bipartite complementation preserves boundedness of clique-width [34].

We need the following lemmas on clique-width, the first one of which is easy to show.

- Lemma 8. The clique-width of a graph of maximum degree at most 2 is at most 4 .

- Lemma 9 ([23]). Let $H$ be a graph. The class of $H$-free graphs has bounded clique-width if and only if $H \subseteq_{i} P_{4}$.

- Lemma 10 ([37]). Let $\left\{H_{1}, \ldots, H_{p}\right\}$ be a finite set of graphs. If $H_{i} \notin \mathcal{S}$ for all $i \in\{1, \ldots, p\}$ then the class of $\left(H_{1}, \ldots, H_{p}\right)$-free graphs has unbounded clique-width.

- Lemma 11 ([15]). Let $G$ be a graph and let $\mathcal{P}$ be the set of all induced subgraphs of $G$ that are prime. Then $\mathrm{cw}(G)=\max _{H \in \mathcal{P}} \mathrm{cw}(H)$. 


\section{The Proof of Theorem 1}

We use the following lemma (proof omitted), which we also need for Theorem 3.

- Lemma 12. If $G$ is a $\left(C_{4}, C_{5}, K_{4}\right)$-free self-complementary graph, then $G \subseteq_{i}$ bull.

We are now ready to prove Theorem 1 . Note that this theorem also holds if $\mathcal{H}$ is infinite.

- Theorem 1 (restated). Let $\mathcal{H}$ be a set of non-empty self-complementary graphs. Then the class of $\mathcal{H}$-free graphs has bounded clique-width if and only if either $P_{1} \in \mathcal{H}$ or $P_{4} \in \mathcal{H}$.

Proof. Suppose there is a graph $H \in \mathcal{H} \cap\left\{P_{1}, P_{4}\right\}$. Then the class of $\mathcal{H}$-free graphs is a subclass of the class of $P_{4}$-free graphs, which have bounded clique-width by Lemma 9 . Now suppose that $\mathcal{H} \cap\left\{P_{1}, P_{4}\right\}=\emptyset$. The only non-empty self-complementary graphs on at most five vertices that are not equal to $P_{1}$ and $P_{4}$ are the bull and the $C_{5}$ (see Figure 2). By Lemma 12, it follows that every graph in $\mathcal{H}$ contains an induced subgraph isomorphic to the bull, $C_{4}, C_{5}$ or $K_{4}$. Therefore the class of $\mathcal{H}$-free graphs contains the class of (bull, $C_{4}, C_{5}, K_{4}$ )-free graphs, which has unbounded clique-width by Lemma 10.

\section{The Proof of Theorem 2}

In this section we prove Theorem 2 by combining known results with the new result that $\left(2 P_{1}+P_{3}, \overline{2 P_{1}+P_{3}}\right)$-free graphs have bounded clique-width. We prove this result in the following way. We first prove two useful structural lemmas, namely Lemmas 13 and 14, which we will use repeatedly throughout the proof. Next, we prove Lemmas 15 and 16, which state that if a $\left(2 P_{1}+P_{3}, \overline{2 P_{1}+P_{3}}\right)$-free graph $G$ contains an induced $C_{5}$ or $C_{6}$, respectively, then $G$ has bounded clique-width. We do this by partitioning the vertices outside this cycle into sets, depending on their neighbourhood in the cycle. We then analyse the edges within these sets and between pairs of such sets. After a lengthy case analysis, we find that $G$ has bounded clique-width in both these cases. By Fact 2 it only remains to analyse $\left(2 P_{1}+P_{3}\right.$, $\left.\overline{2 P_{1}+P_{3}}\right)$-free graphs that are also $\left(C_{5}, C_{6}, \overline{C_{6}}\right)$-free. Next, in Lemma 17 , we show that if such graphs are prime, then they are either $K_{7}$-free or $\overline{K_{7}}$-free. In Lemma 27 we use the fact that $\left(2 P_{1}+P_{3}, \overline{2 P_{1}+P_{3}}\right)$-free graphs are $\chi$-bounded to deal with the case where a graph in the class is $K_{7}$-free. Finally, we combine all these results together to obtain the new result (Theorem 28). We omit the proofs of Lemmas 13-16.

- Lemma 13. Let $G$ be a $\left(2 P_{1}+P_{3}, \overline{2 P_{1}+P_{3}}\right)$-free graph whose vertex set can be partitioned into two sets $X$ and $Y$, each of which is a clique or an independent set. Then by deleting at most one vertex from each of $X$ and $Y$, it is possible to obtain subsets such that the edges between them form a matching or a co-matching.

- Lemma 14. Let $G$ be a $\left(2 P_{1}+P_{3}, \overline{2 P_{1}+P_{3}}\right)$-free graph whose vertex set can be partitioned into a clique $X$ and an independent set $Y$. Then by deleting at most three vertices from each of $X$ and $Y$, it is possible to obtain subsets that are either complete or anti-complete to each other.

- Lemma 15. The class of $\left(2 P_{1}+P_{3}, \overline{2 P_{1}+P_{3}}\right)$-free graphs containing an induced $C_{5}$ has bounded clique-width.

- Lemma 16. The class of $\left(2 P_{1}+P_{3}, \overline{2 P_{1}+P_{3}}\right)$-free graphs containing an induced $C_{6}$ has bounded clique-width.

- Lemma 17. Every prime $\left(2 P_{1}+P_{3}, \overline{2 P_{1}+P_{3}}, C_{6}, \overline{C_{6}}\right)$-free graph is $K_{7}$-free or $\overline{K_{7}}$-free. 
Proof. Let $G$ be a prime $\left(2 P_{1}+P_{3}, \overline{2 P_{1}+P_{3}}, C_{6}, \overline{C_{6}}\right)$-free graph. Suppose, for contradiction, that $G$ contains an induced $K_{7}$ and an induced $\overline{K_{7}}$. We will show that in this case the graph $G$ is not prime. Note that any induced $K_{7}$ and induced $\overline{K_{7}}$ in $G$ can share at most one vertex. We may therefore assume that $G$ contains a clique $C$ on at least six vertices and a vertex-disjoint independent set $I$ on at least six vertices. Furthermore, we may assume that $C$ is a maximum clique in $G \backslash I$ and $I$ is a maximum independent set in $G \backslash C$ (if not, then replace $C$ or $I$ with a bigger clique or independent set, respectively).

By Lemma 14, there exist sets $R_{1} \subset C$ and $R_{2} \subset I$ each of size at most 3 such that $C^{\prime}=C \backslash R_{1}$ is either complete or anti-complete to $I^{\prime}=I \backslash R_{2}$. Without loss of generality, we may assume that $R_{1}$ and $R_{2}$ are minimal, in the sense that the above property does not hold if we remove any vertex from $R_{1}$ or $R_{2}$. Note that the class of prime $\left(2 P_{1}+P_{3}, \overline{2 P_{1}+P_{3}}, C_{6}, \overline{C_{6}}\right)$ free graphs containing an induced $K_{7}$ and an induced $\overline{K_{7}}$ is closed under complementation. Therefore, complementing $G$ if necessary (in which case the sets $I$ and $C$ will be swapped, and the sets $R_{1}$ and $R_{2}$ will be swapped), we may assume that $C^{\prime}$ is anti-complete to $I^{\prime}$.

- Claim 18. $\left|R_{1}\right| \leq 1$ and $\left|R_{2}\right| \leq 1$.

By construction, $R_{1}$ and $R_{2}$ each contain at most three vertices and $I^{\prime}$ and $C^{\prime}$ each contain at least three vertices. Since $R_{1}$ (resp. $R_{2}$ ) is minimal, every vertex of $R_{1}$ (resp. $R_{2}$ ) has at least one neighbour in $I^{\prime}$ (resp. $C^{\prime}$ ).

Choose $i_{1}, i_{2} \in I^{\prime}$ arbitrarily and suppose, for contradiction, that $y \in R_{2}$ is not complete to $C^{\prime}$. Then $y$ must have a neighbour $c_{1} \in C^{\prime}$ and a non-neighbour $c_{2} \in C^{\prime}$, so $G\left[i_{1}, i_{2}, y, c_{1}, c_{2}\right]$ is a $2 P_{1}+P_{3}$, a contradiction. Therefore $R_{2}$ is complete to $C^{\prime}$. If $y, y^{\prime} \in R_{2}$ then for arbitrary $c_{1} \in C^{\prime}$, the graph $G\left[i_{1}, i_{2}, y, c_{1}, y^{\prime}\right]$ is a $2 P_{1}+P_{3}$, a contradiction. It follows that $\left|R_{2}\right| \leq 1$.

Choose $c_{1}, c_{2} \in C^{\prime}$ arbitrarily. Suppose, for contradiction, that $x \in R_{1}$ has two nonneighbours $i_{1}, i_{2} \in I^{\prime}$. Recall that $x$ must have a neighbour $i_{3} \in I^{\prime}$, so $G\left[i_{1}, i_{2}, i_{3}, x, c_{1}\right]$ is a $2 P_{1}+P_{3}$, a contradiction. Therefore every vertex of $R_{1}$ has at most one non-neighbour in $I^{\prime}$. Suppose, for contradiction, that $x, x^{\prime} \in R_{1}$. Since $I^{\prime}$ contains at least three vertices, there must be a vertex $i_{1} \in I^{\prime}$ that is a common neighbour of $x$ and $x^{\prime}$. Now $G\left[x, x^{\prime}, c_{1}, i_{1}, c_{2}\right]$ is a $\overline{2 P_{1}+P_{3}}$, a contradiction. It follows that $\left|R_{1}\right| \leq 1$. This completes the proof of Claim 18 .

Note that Claim 18 implies that $\left|C^{\prime}\right| \geq 5$ and $\left|I^{\prime}\right| \geq 5$. Let $A$ be the set of vertices in $V \backslash(C \cup I)$ that are complete to $C^{\prime}$. If $x \in A$ is adjacent to $y \in R_{1}$ then by Claim 18 $C \cup\{x\}$ is a bigger clique than $C$, contradicting the maximality of $C$. It follows that $A$ is anti-complete to $R_{1}$. If $x, y \in A$ are adjacent then by Claim 18, $(C \cup\{x, y\}) \backslash R_{1}$ is a bigger clique than $C$, contradicting the maximality of $C$. It follows that $A$ is an independent set. Furthermore, by the maximality of $I$ and the definition of $A$, every vertex in $V \backslash(C \cup I \cup A)$ has a neighbour in $I$ and non-neighbour in $C^{\prime}$.

- Claim 19. Let $x$ be a vertex in $V \backslash(C \cup I \cup A)$. Then either $x$ is complete to $I^{\prime}$, or $x$ has exactly one neighbour in $I$.

Suppose, for contradiction, that $x$ has a non-neighbour $z$ in $I^{\prime}$, and two neighbours $y, y^{\prime} \in I$. Now $x$ cannot have another non-neighbour $z^{\prime} \in I \backslash\{z\}$, otherwise $G\left[z, z^{\prime}, y, x, y^{\prime}\right]$ would be a $2 P_{1}+P_{3}$. Therefore $x$ must be complete to $I \backslash\{z\}$. In particular, since $\left|I^{\prime}\right| \geq 5$, this means that $x$ has two neighbours in $I^{\prime}$, say $y_{1}$ and $y_{2}$ (not necessarily distinct from $y$ and $y^{\prime}$ ). Recall that $x$ must have a non-neighbour $c_{1} \in C^{\prime}$. Now $G\left[c_{1}, z, y_{1}, x, y_{2}\right]$ is a $2 P_{1}+P_{3}$. This contradiction completes the proof of Claim 19.

By Claim 19 we can partition the vertex set $V \backslash(C \cup I \cup A)$ into subsets $V_{I^{\prime}}$ and $V_{x}$ for every $x \in I$, where $V_{I^{\prime}}$ is the set of vertices that are complete to $I^{\prime}$, and $V_{x}$ is the set of vertices whose unique neighbour in $I$ is $x$. Let $U_{x}=V_{x} \cup\{x\}$. 
- Claim 20. For all $x \in I^{\prime}, U_{x}$ is anti-complete to $C^{\prime}$.

Suppose $x \in I^{\prime}$. Clearly $x$ is anti-complete to $C^{\prime}$. Suppose, for contradiction, that $y \in$ $U_{x} \backslash\{x\}=V_{x}$ has a neighbour $z \in C^{\prime}$ and choose $u, v \in I^{\prime} \backslash\{x\}$. Then $G[u, v, x, y, z]$ is a $2 P_{1}+P_{3}$. This contradiction completes the proof of Claim 20 .

Claim 21. For every $x \in I$, the set $U_{x}$ is a clique.

Note that $x \in I$ is adjacent to all other vertices of $U_{x}$, by definition. If $y, z \in V_{x}$ are nonadjacent then $(I \backslash\{x\}) \cup\{y, z\}$ would be a bigger independent set than $I$, a contradiction.

- Claim 22. If $x, y \in I$ are distinct, then $U_{x}$ is anti-complete to $U_{y}$.

Clearly $x$ is anti-complete to $U_{y}$ and $y$ is anti-complete to $U_{x}$. Suppose, for contradiction, that $x^{\prime} \in U_{x} \backslash\{x\}$ is adjacent to $y^{\prime} \in U_{y} \backslash\{y\}$. Choose $u, v \in I \backslash\{x, y\}$. Then $G\left[u, v, x, x^{\prime}, y^{\prime}\right]$ is a $2 P_{1}+P_{3}$. This contradiction completes the proof of Claim 22 .

- Claim 23. For every $x \in I^{\prime}$, the set $U_{x}$ is complete to $V_{I^{\prime}}$.

By definition, $x$ is complete to $V_{I^{\prime}}$. Suppose, for contradiction that $x^{\prime} \in U_{x} \backslash\{x\}$ is nonadjacent to $y \in V_{I^{\prime}}$. As $y \notin A$, the vertex $y$ must have a non-neighbour $c_{1} \in C^{\prime}$ and note that $x^{\prime}$ is non-adjacent to $c_{1}$ by Claim 20. Choose $u, v \in I^{\prime} \backslash\{x\}$. Then $G\left[c_{1}, x^{\prime}, u, y, v\right]$ is a $2 P_{1}+P_{3}$. This contradiction proves Claim 23 .

Suppose $x \in I^{\prime}$. Claim 21 implies that $U_{x}$ is a clique, Claim 20 that $U_{x}$ is anti-complete to $C^{\prime}$ and Claim 23 that $U_{x}$ is complete to $V_{I^{\prime}}$. Furthermore for all $y \in I \backslash\{x\}$, Claim 22 implies that $U_{x}$ is anti-complete to $U_{y}$. We conclude that given any two vertices $x, y \in I^{\prime}$, no vertex in $V \backslash\left(A \cup R_{1} \cup U_{x} \cup U_{y}\right)$ can distinguish the set $U_{x} \cup U_{y}$. In the remainder of the proof, we will show there exist $x, y \in I^{\prime}$ such that no vertex of $A \cup R_{1}$ distinguishes the set $U_{x} \cup U_{y}$, meaning that $U_{x} \cup U_{y}$ is a non-trivial module, contradicting the assumption that $G$ is prime.

- Claim 24. If $u \in A \cup R_{1}$ then either $u$ is anti-complete to $U_{x}$ for all $x \in I^{\prime}$ or else $u$ is complete to $U_{x}$ for all but at most one $x \in I^{\prime}$.

Suppose, for contradiction, that the claim does not hold for a vertex $u \in A \cup R_{1}$. Then $u$ must have a neighbour $x^{\prime} \in U_{x}$ for some $x \in I^{\prime}$ and must have non-neighbours $y^{\prime} \in U_{y}$ and $z^{\prime} \in U_{z}$ for some $y, z \in I^{\prime}$ with $y \neq z$. Since $\left|I^{\prime}\right| \geq 5$, we may also assume that $x \notin\{y, z\}$. Choose $c_{1} \in C^{\prime}$ arbitrarily. By Claim 20, $c_{1}$ is non-adjacent to $x^{\prime}, y^{\prime}$ and $z^{\prime}$. It follows that $G\left[y^{\prime}, z^{\prime}, c_{1}, u, x^{\prime}\right]$ is a $2 P_{1}+P_{3}$. This contradiction completes the proof of Claim 24 .

Let $A^{*}$ denote the set of vertices in $A \cup R_{1}$ that have a neighbour in $U_{x}$ for some $x \in I^{\prime}$.

- Claim 25. The set $A^{*}$ is complete to all, except possibly two, sets $U_{x}, x \in I^{\prime}$.

Suppose, for contradiction, that there are three different vertices $x, y, z \in I^{\prime}$ such that $A^{*}$ is complete to none of the sets $U_{x}, U_{y}$, and $U_{z}$. By Claim 24 and the definition of $A^{*}$, every vertex in $A^{*}$ is complete to at least two of the sets $U_{x}, U_{y}, U_{z}$. Therefore there exist three vertices $u, v, w \in A^{*}$ such that:

- $u$ is not adjacent to some vertex $x^{\prime} \in U_{x}$, but is complete to $U_{y}$ and $U_{z}$;

- $v$ is not adjacent to some vertex $y^{\prime} \in U_{y}$, but is complete to $U_{x}$ and $U_{z}$;

- $w$ is not adjacent to some vertex $z^{\prime} \in U_{z}$, but is complete to $U_{x}$ and $U_{y}$. 
Therefore $G\left[u, y^{\prime}, w, x^{\prime}, v, z^{\prime}\right]$ is a $C_{6}$. This contradiction completes the proof of Claim 25 .

Now, as $\left|I^{\prime}\right| \geq 5$, Claims 24 and 25 imply there exist two distinct vertices $x, y \in I^{\prime}$ such that every vertex of $A \cup R_{1}$ is either complete or anti-complete to $U_{x} \cup U_{y}$. Hence $U_{x} \cup U_{y}$ is a non-trivial module in $G$, contradicting the fact that $G$ is prime. This completes the proof.

The chromatic number $\chi(G)$ of a graph $G$ is the minimum positive integer $k$ such that $G$ is $k$-colourable. The clique number $\omega(G)$ of $G$ is the size of a largest clique in $G$. A class $\mathcal{C}$ of graphs is $\chi$-bounded if there is a function $f$ such that $\chi(G) \leq f(\omega(G))$ for all $G \in \mathcal{C}$.

- Lemma 26 ([32]). For every natural number $k$ the class of $P_{k}$-free graphs is $\chi$-bounded.

- Lemma 27. For $k \geq 1,\left(K_{k}, 2 P_{1}+P_{3}, \overline{2 P_{1}+P_{3}}\right)$-free graphs have bounded clique-width.

Proof. Fix a constant $k \geq 1$ and let $G$ be a $\left(K_{k}, 2 P_{1}+P_{3}, \overline{2 P_{1}+P_{3}}\right)$-free graph. By Lemma 16, we may assume that $G$ is $C_{6}$-free. Since $G$ is $\left(2 P_{1}+P_{3}\right)$-free, it is $P_{7}$-free, so by Lemma 26 it has chromatic number at most $\ell$ for some constant $\ell$. This means that we can partition the vertices of $G$ into $\ell$ independent sets $V_{1}, \ldots, V_{\ell}$ (some of which may be empty). By Lemma 13, deleting finitely many vertices (which we may do by Fact 2), we may assume that for all distinct $i, j \in\{1, \ldots, \ell\}$, the edges between $V_{i}$ and $V_{j}$ form a matching or a co-matching. Since $G$ is $C_{6}$-free, if the vertices between $V_{i}$ and $V_{j}$ form co-matching, this co-matching can contain at most two non-edges. Therefore, by deleting finitely many vertices (which we may do by Fact 2), we may assume that the edges between $V_{i}$ and $V_{j}$ form a matching or $V_{i}$ and $V_{j}$ are complete to each other. By deleting finitely many vertices (which we may do by Fact 2), we may assume that each set $V_{i}$ is either empty or contains at least five vertices.

Suppose the edges from $V_{i}$ to $V_{j}$ and the edges from $V_{i}$ to $V_{k}$ form a matching and that there is a vertex $x \in V_{i}$ that has a neighbour $y \in V_{j}$ and a neighbour $z \in V_{k}$. Then $y$ must be adjacent to $z$, otherwise for $x^{\prime}, x^{\prime \prime} \in V_{i} \backslash\{x\}$ the graph $G\left[x^{\prime}, x^{\prime \prime}, y, x, z\right]$ would be a $2 P_{1}+P_{3}$, a contradiction. If $V_{j}$ is complete to $V_{k}$ then for $y^{\prime}, y^{\prime \prime} \in V_{j}, z^{\prime} \in V_{k}$ and $x^{\prime}, x^{\prime \prime} \in V_{i} \backslash\left(N\left(y^{\prime}\right) \cup N\left(y^{\prime \prime}\right) \cup N\left(z^{\prime}\right)\right)$ (such vertices exist since each of $y^{\prime}, y^{\prime \prime}$ and $z^{\prime}$ have at most one neighbour in $V_{i}$ and $V_{i}$ contains at least five vertices) we have $G\left[x^{\prime}, x^{\prime \prime}, y^{\prime}, z^{\prime}, y^{\prime \prime}\right]$ is a $2 P_{1}+P_{3}$, a contradiction. Therefore the edges between $V_{j}$ and $V_{k}$ form a matching.

Now for each $i, j \in\{1, \ldots, \ell\}$ with $i<j$, if $V_{i}$ is complete to $V_{j}$, then by Fact 2 we may apply a bipartite complementation between $V_{i}$ and $V_{j}$. Let $G^{\prime}$ be the resulting graph. The previous paragraph implies if $x$ has two neighbours $y$ and $z$ in $G^{\prime}$ then $y$ is adjacent to $z$ in $G$, so $G^{\prime}$ is $P_{3}$-free. So $G^{\prime}$ is a disjoint union of cliques, and thus has clique-width at most 2.

We are now ready to prove our main result.

- Theorem 28. The class of $\left(2 P_{1}+P_{3}, \overline{2 P_{1}+P_{3}}\right)$-free graphs has bounded clique-width.

Proof. Let $G$ be a $\left(2 P_{1}+P_{3}, \overline{2 P_{1}+P_{3}}\right)$-free graph. By Lemma 11, we may assume that $G$ is prime. If $G$ contains an induced $C_{6}$ then we are done by Lemma 16. If $G$ contains an induced $\overline{C_{6}}$ then we are done by Lemma 16 and Fact 2 . We may therefore assume that $G$ is also $\left(C_{6}, \overline{C_{6}}\right)$-free. By Lemma 17 , we may assume that $G$ is either $K_{7}$-free or $\overline{K_{7}}$-free. By Fact 2, we may assume that $G$ is $K_{7}$-free. Lemma 27 completes the proof.

Combining Theorem 28 with the current state-of-the-art for classifying the boundedness of clique-width for $\left(H_{1}, H_{2}\right)$-free graphs (see [20]) yields Theorem 2 (proof omitted).

- Theorem 2 (restated). For a graph $H$, the class of $(H, \bar{H})$-free graphs has bounded cliquewidth if and only if $H$ or $\bar{H}$ is an induced subgraph of $K_{1,3}, P_{1}+P_{4}, 2 P_{1}+P_{3}$ or $s P_{1}$ for some $s \geq 1$. 

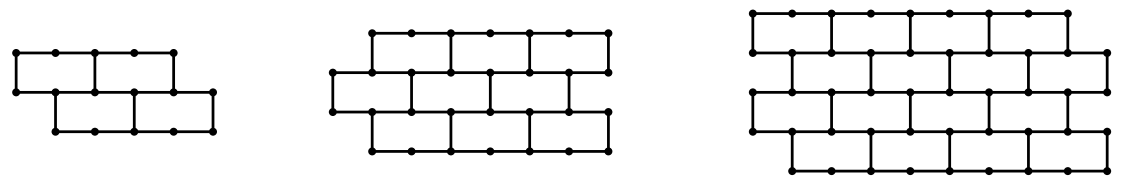

Figure 4 Walls of height 2, 3 and 4, respectively.

\section{$5 \quad$ New Classes of Unbounded Clique-Width and Proof of Theorem 3}

In this section we first identify three new graph classes of unbounded clique-width. To do so, we need the notion of a wall. Figure 4 shows three walls of different height (see e.g. [12] for a formal definition). The class of walls is well known to have unbounded clique-width; see for example [34]. A $k$-subdivided wall is the graph obtained from a wall after subdividing each edge exactly $k$ times for some constant $k \geq 0$. The following lemma is well known.

- Lemma 29 ([37]). Let $k \geq 0$. The class of $k$-subdivided walls has unbounded clique-width.

Dabrowski et al. [17] showed that $\left(4 P_{1}, \overline{3 P_{1}+P_{2}}\right)$-free graphs have unbounded cliquewidth. However, their construction was not $C_{5}$-free. We give an alternative construction that neither contains an induced $C_{5}$ nor an induced copy of any larger self-complementary graph. Namely, we first consider a graph $H^{\prime}$ that is a 1-subdivided wall. By Lemma 29, such graphs have unbounded clique-width. Let $V_{1}$ be the set of vertices in $H^{\prime}$ that are also present in $H$. Let $V_{2}$ be the set of vertices obtained from subdividing vertical edges in $H$, and let $V_{3}$ be the set of vertices obtained from subdividing horizontal edges. Note that $V_{1}, V_{2}$ and $V_{3}$ are independent sets. Furthermore, every vertex in $V_{1}$ has at most one neighbour in $V_{2}$ and at most two neighbours in $V_{3}$, while every vertex in $V_{2} \cup V_{3}$ has at most two neighbours, each of which is in $V_{1}$. Let $H^{\prime \prime}$ be the graph obtained from $H^{\prime}$ by applying complementations on $V_{1}, V_{2}$ and $V_{3}$. By Fact 2, such graphs have unbounded clique-width. We claim that $H^{\prime \prime}$ is $\left(\left\{4 P_{1}, \overline{3 P_{1}+P_{2}}\right\} \cup \mathcal{F}\right)$-free, where $\mathcal{F}$ is the set of all self-complementary graphs on at least five vertices that are not equal to the bull (proof omitted). This leads to the following theorem.

- Theorem 30. Let $\mathcal{F}$ be the set of all self-complementary graphs on at least five vertices that are not equal to the bull. The class of $\left(\left\{4 P_{1}, \overline{3 P_{1}+P_{2}}\right\} \cup \mathcal{F}\right)$-free graphs has unbounded clique-width.

By Lemma 12, any self-complementary graph on at least five vertices not equal to the bull has an induced subgraph isomorphic to $C_{4}, C_{5}$ or $K_{4}$, so such graphs are automatically excluded from the class specified in our next theorem. Its proof, which we omitted, is based on observing that the construction of Brandstädt et al. [7] for proving that $\left(C_{4}, K_{1,3}, K_{4}, \overline{2 P_{1}+P_{2}}\right)$-free graphs have unbounded clique-width is, in fact, also $C_{5}$-free.

- Theorem 31. $\left(C_{4}, C_{5}, K_{1,3}, K_{4}, \overline{2 P_{1}+P_{2}}\right)$-free graphs have unbounded clique-width.

For our third result we need two lemmas. Given natural numbers $k, \ell$, let $R b(k, \ell)$ denote the smallest number such that if every edge of a $K_{R b(k, \ell), R b(k, \ell)}$ is coloured red or blue then it will contain a monochromatic $K_{k, \ell}$. It is known that $R b(k, \ell)$ always exists [1].

- Lemma $32([1]) . \quad R b(2,2)=5$.

Let $G=(V, E)$ be a split graph. By definition, $G$ has a split partition, that is, a partition of $V$ into two (possibly empty) sets $C$ and $I$, where $C$ is a clique and $I$ is an independent set. A split graph $G$ may have multiple split partitions. For self-complementary split graphs we can show the following (proof omitted). 
- Lemma 33. Let $G$ be a self-complementary split graph on $n$ vertices. If $n$ is even, then $G$ has a unique split partition and in this partition the clique and independent set are of equal size. If $n$ is odd, then there exists a vertex $v$ such that $G \backslash v$ is also a self-complementary split graph.

- Theorem 34. Let $\mathcal{F}$ be the set of all self-complementary graphs on at least five vertices that are not equal to the bull. The class of $\left(\left\{C_{4}, 2 P_{2}\right\} \cup \mathcal{F}\right)$-free graphs has unbounded clique-width.

Proof. First note that the only self-complementary graph on five vertices apart from the bull is the $C_{5}$. Since $C_{5} \in \mathcal{F}$, by Lemma 6 , we may remove all graphs that are not split from $\mathcal{F}$, apart from $C_{5}$; in particular, this means that we remove $X_{4}, \ldots, X_{10}$ from $\mathcal{F}$ (see also Figure 3). By Lemma 33, if $G \in \mathcal{F}$ has an odd number of vertices, but is not equal to $C_{5}$, then $G \backslash v \in \mathcal{F}$ for some vertex $v \in V(G)$. Let $\mathcal{F}^{\prime}$ be the set of self-complementary split graphs on at least eight vertices that have an even number of vertices. It follows that the class of $\mathcal{F}^{\prime}$-free split graphs is equal to the class of $\left(\left\{C_{4}, 2 P_{2}\right\} \cup \mathcal{F}\right)$-free graphs.

Consider a 2-subdivided wall $H$ and note that it is $\left(C_{4}, C_{8}\right)$-free; recall that 2-subdivided walls have unbounded clique-width by Lemma 29. Note that $H$ is a bipartite graph, and fix a bipartition $(A, B)$ of $H$. Let $H^{\prime}$ be the graph obtained from $H$ by applying a complementation to $A$ and note that $H^{\prime}$ is a split graph. In $H^{\prime}$, every vertex in $B$ has a non-neighbour in $A$ and every vertex in $A$ has a neighbour in $B$, so $(A, B)$ is the unique split partition of $H^{\prime}$. By Fact 2, the family of graphs $H^{\prime}$ produced in this way also has unbounded clique-width. It remains to show that $H^{\prime}$ is $\mathcal{F}^{\prime}$-free.

First note that $X_{1}$ (see also Figure 3 ) is the graph obtained from the bipartite graph $C_{8}$ by complementing one of the independent sets in the bipartition. Since $H$ is $C_{8}$-free and $X_{1}$ has a unique split partition (by Lemma 33), it follows that $H^{\prime}$ is $X_{1}$-free. Note that $H$ is $C_{4}$-free and so $H^{\prime}$ does not contain two vertices $x, x^{\prime}$ in the clique $A$ and two vertices $y, y^{\prime}$ in the independent set $B$ such that $\left\{x, x^{\prime}\right\}$ is complete to $\left\{y, y^{\prime}\right\}$. Now suppose $G \in \mathcal{F}^{\prime} \backslash\left\{X_{1}\right\}$. Recall that by Lemma $33, G$ has a unique split partition $(C, I)$, and this partition has the property that $|C|=|I|$. Therefore, if we can show that $G$ contains two vertices $x, x^{\prime} \in C$ and two vertices $y, y^{\prime} \in I$ with $\left\{x, x^{\prime}\right\}$ complete to $\left\{y, y^{\prime}\right\}$ then $H^{\prime}$ must be $G$-free and the proof is complete. It is easy to verify that this is the case if $G \in\left\{X_{2}, X_{3}\right\}$ (see also Figure 3 and recall that $\left.X_{4}, \ldots, X_{10} \notin \mathcal{F}^{\prime}\right)$. Otherwise, $G$ has at least ten vertices so $|C|,|I| \geq 5$. By Lemma 32 , there must be two vertices $x, x^{\prime} \in C$ and two vertices $y, y^{\prime} \in I$ with $\left\{x, x^{\prime}\right\}$ either complete or anti-complete to $\left\{y, y^{\prime}\right\}$. In the first case we are done. In the second case we note that complementing $G$ will swap the sets $C$ and $I$ and make $\left\{x, x^{\prime}\right\}$ complete to $\left\{y, y^{\prime}\right\}$, returning us to the previous case. We conclude that $H^{\prime}$ is indeed $\mathcal{F}^{\prime}$-free.

We are now ready to prove Theorem 3 . Note that this theorem holds even if $\mathcal{F}$ is infinite.

- Theorem 3 (restated). Let $\mathcal{F}$ be a set of self-complementary graphs on at least five vertices not equal to the bull. For a graph $H$, the class of $(\{H, \bar{H}\} \cup \mathcal{F})$-free graphs has bounded clique-width if and only if $H$ or $\bar{H}$ is an induced subgraph of $K_{1,3}, P_{1}+P_{4}, 2 P_{1}+P_{3}$ or $s P_{1}$ for some $s \geq 1$.

Proof. Let $H$ be a graph. By Theorem 2, if $H$ or $\bar{H}$ is an induced subgraph of $K_{1,3}$, $P_{1}+P_{4}, 2 P_{1}+P_{3}$ or $s P_{1}$ for some $s \geq 1$, then the class of $(\{H, \bar{H}\} \cup \mathcal{F})$-free graphs has bounded clique-width. Consider a graph $F \in \mathcal{F}$. Since $F$ contains at least five vertices and is not isomorphic to the bull, Lemma 12 implies that $F$ contains an induced subgraph isomorphic to $C_{4}, C_{5}$ or $K_{4}$, and so $F \notin \mathcal{S}$. Therefore the class of $(\{H, \bar{H}\} \cup \mathcal{F})$-free graphs contains the class of $\left(H, \bar{H}, C_{4}, C_{5}, K_{4}\right)$-free graphs. If $H \notin \mathcal{S}$ and $\bar{H} \notin \mathcal{S}$, then the class of $\left(H, \bar{H}, C_{4}, C_{5}, K_{4}\right)$-free graphs has unbounded clique-width by Lemma 10. By Fact 2, we 
may therefore assume that $H \in \mathcal{S}$. By Lemma 7 , we may assume $H$ contains $K_{1,3}+P_{1}, 2 P_{2}$, $3 P_{1}+P_{2}$ or $S_{1,1,2}$ as an induced subgraph, otherwise we are done. In this case, the class of $(\{H, \bar{H}\} \cup \mathcal{F})$-free graphs contains the class of $\left(K_{1,3}, K_{4}, C_{4}, C_{5}\right)$-free, $\left(\left\{2 P_{2}, C_{4}\right\} \cup \mathcal{F}\right)$-free, $\left(\left\{4 P_{1}, \overline{3 P_{1}+P_{2}}\right\} \cup \mathcal{F}\right)$-free or $\left(K_{1,3}, \overline{2 P_{1}+P_{2}}, C_{4}, C_{5}, K_{4}\right)$-free graphs, respectively. These classes have unbounded clique-width by Theorems $31,34,30$ and 31 , respectively. This completes the proof.

\section{References}

1 Lowell W. Beineke and Allen J. Schwenk. On a bipartite form of the Ramsey problem. Congressus Numerantium, XV:17-22, 1975.

2 Alexandre Blanché, Konrad K. Dabrowski, Matthew Johnson, and Daniël Paulusma. Hereditary graph classes: When the complexities of Colouring and Clique Cover coincide. CoRR, abs/1607.06757, 2016.

3 Rodica Boliac and Vadim V. Lozin. On the clique-width of graphs in hereditary classes. Proc. ISAAC 2002, LNCS, 2518:44-54, 2002.

4 Andreas Brandstädt, Konrad K. Dabrowski, Shenwei Huang, and Daniël Paulusma. Bounding the clique-width of $H$-free split graphs. Discrete Applied Mathematics, 211:30-39, 2016.

5 Andreas Brandstädt, Konrad K. Dabrowski, Shenwei Huang, and Daniël Paulusma. Bounding the clique-width of $H$-free chordal graphs. Journal of Graph Theory, (in press), 2017.

6 Andreas Brandstädt, Feodor F. Dragan, Hoàng-Oanh Le, and Raffaele Mosca. New graph classes of bounded clique-width. Theory of Computing Systems, 38(5):623-645, 2005.

7 Andreas Brandstädt, Joost Engelfriet, Hoàng-Oanh Le, and Vadim V. Lozin. Clique-width for 4-vertex forbidden subgraphs. Theory of Computing Systems, 39(4):561-590, 2006.

8 Andreas Brandstädt, Tilo Klembt, and Suhail Mahfud. $P_{6^{-}}$and triangle-free graphs revisited: structure and bounded clique-width. Discrete Mathematics and Theoretical Computer Science, 8(1):173-188, 2006.

9 Andreas Brandstädt, Hoàng-Oanh Le, and Raffaele Mosca. Gem- and co-gem-free graphs have bounded clique-width. International Journal of Foundations of Computer Science, 15(1):163-185, 2004.

10 Andreas Brandstädt, Hoàng-Oanh Le, and Raffaele Mosca. Chordal co-gem-free and $\left(P_{5}\right.$, gem $)$-free graphs have bounded clique-width. Discrete Applied Mathematics, 145(2):232-241, 2005.

11 Andreas Brandstädt and Suhail Mahfud. Maximum weight stable set on graphs without claw and co-claw (and similar graph classes) can be solved in linear time. Information Processing Letters, 84(5):251-259, 2002.

12 Julia Chuzhoy. Improved bounds for the flat wall theorem. Proc. SODA 2015, pages 256-275, 2015.

13 Derek G. Corneil, Michel Habib, Jean-Marc Lanlignel, Bruce A. Reed, and Udi Rotics. Polynomial-time recognition of clique-width $\leq 3$ graphs. Discrete Applied Mathematics, 160(6):834-865, 2012.

14 Bruno Courcelle, Johann A. Makowsky, and Udi Rotics. Linear time solvable optimization problems on graphs of bounded clique-width. Theory of Computing Systems, 33(2):125-150, 2000.

15 Bruno Courcelle and Stephan Olariu. Upper bounds to the clique width of graphs. Discrete Applied Mathematics, 101(1-3):77-114, 2000.

16 Konrad K. Dabrowski, François Dross, and Daniël Paulusma. Colouring diamond-free graphs. Journal of Computer and System Sciences, (to appear).

17 Konrad K. Dabrowski, Petr A. Golovach, and Daniël Paulusma. Colouring of graphs with Ramsey-type forbidden subgraphs. Theoretical Computer Science, 522:34-43, 2014. 
18 Konrad K. Dabrowski, Shenwei Huang, and Daniël Paulusma. Bounding clique-width via perfect graphs. Journal of Computer and System Sciences, (in press).

19 Konrad K. Dabrowski, Vadim V. Lozin, and Daniël Paulusma. Clique-width and well-quasi ordering of triangle-free graph classes. Proc. WG 2017, LNCS, (to appear).

20 Konrad K. Dabrowski, Vadim V. Lozin, and Daniël Paulusma. Well-quasi-ordering versus clique-width: New results on bigenic classes. Order, (to appear).

21 Konrad K. Dabrowski, Vadim V. Lozin, Rajiv Raman, and Bernard Ries. Colouring vertices of triangle-free graphs without forests. Discrete Mathematics, 312(7):1372-1385, 2012.

22 Konrad K. Dabrowski and Daniël Paulusma. Classifying the clique-width of $H$-free bipartite graphs. Discrete Applied Mathematics, 200:43-51, 2016.

23 Konrad K. Dabrowski and Daniël Paulusma. Clique-width of graph classes defined by two forbidden induced subgraphs. The Computer Journal, 59(5):650-666, 2016.

24 H. N. de Ridder et al. Information System on Graph Classes and their Inclusions, 20012013. http://www.graphclasses.org.

25 Wolfgang Espelage, Frank Gurski, and Egon Wanke. How to solve NP-hard graph problems on clique-width bounded graphs in polynomial time. Proc. WG 2001, LNCS, 2204:117-128, 2001.

26 Alastair Farrugia. Self-complementary graphs and generalisations: a comprehensive reference manual. Master's thesis, University of Malta, 1999.

27 Michael R. Fellows, Frances A. Rosamond, Udi Rotics, and Stefan Szeider. Clique-width is NP-Complete. SIAM Journal on Discrete Mathematics, 23(2):909-939, 2009.

28 Stéphane Földes and Peter Ladislaw Hammer. Split graphs. Congressus Numerantium, XIX:311-315, 1977.

29 Petr A. Golovach, Matthew Johnson, Daniël Paulusma, and Jian Song. A survey on the computational complexity of colouring graphs with forbidden subgraphs. Journal of Graph Theory, 84(4):331-363, 2017.

30 Martin Grohe and Pascal Schweitzer. Isomorphism testing for graphs of bounded rank width. Proc. FOCS 2015, pages 1010-1029, 2015.

31 Frank Gurski. The behavior of clique-width under graph operations and graph transformations. Theory of Computing Systems, 60(2):346-376, 2017.

32 András Gyárfás. Problems from the world surrounding perfect graphs. Applicationes Mathematicae, 19(3-4):413-441, 1987.

33 Öjvind Johansson. Clique-decomposition, NLC-decomposition, and modular decomposition - relationships and results for random graphs. Congressus Numerantium, 132:39-60, 1998.

34 Marcin Kamiński, Vadim V. Lozin, and Martin Milanič. Recent developments on graphs of bounded clique-width. Discrete Applied Mathematics, 157(12):2747-2761, 2009.

35 Daniel Kobler and Udi Rotics. Edge dominating set and colorings on graphs with fixed clique-width. Discrete Applied Mathematics, 126(2-3):197-221, 2003.

36 Vadim V. Lozin and Dieter Rautenbach. On the band-, tree-, and clique-width of graphs with bounded vertex degree. SIAM Journal on Discrete Mathematics, 18(1):195-206, 2004.

37 Vadim V. Lozin and Dieter Rautenbach. The tree- and clique-width of bipartite graphs in special classes. Australasian Journal of Combinatorics, 34:57-67, 2006.

38 Johann A. Makowsky and Udi Rotics. On the clique-width of graphs with few $P_{4}$ 's. International Journal of Foundations of Computer Science, 10(03):329-348, 1999.

39 Sang-Il Oum and Paul D. Seymour. Approximating clique-width and branch-width. Journal of Combinatorial Theory, Series B, 96(4):514-528, 2006.

40 Michaël Rao. MSOL partitioning problems on graphs of bounded treewidth and cliquewidth. Theoretical Computer Science, 377(1-3):260-267, 2007.

41 Ronald C. Read. On the number of self-complementary graphs and digraphs. Journal of the London Mathematical Society, s1-38(1):99-104, 1963. 